\title{
GROUPS WITH LARGE CONJUGACY CLASSES
}

\author{
BOLA O. BALOGUN \\ (Received 28 January 1976; revised 7 September 1976) \\ Communicated by M. F. Newman
}

\begin{abstract}
A finite group is called repetition-free if its conjugacy classes have distinct sizes. It is known that a supersolvable repetition-free group is necessarily isomorphic to $\mathrm{Sym}(3)$. the symmetric group on three symbols. Thus the question arises as to whether Sym (3) is the only repetition-free group. In this paper it is proved that if $m_{k}$ denotes the minimum of the orders of the centralizers of elements of a repetition-free group $G$ and $m_{\star} \leqq 4$ then $G$ is isomorphic to Sym (3).
\end{abstract}

\section{Introduction}

Let $G$ be a finite group. Let $k=k(G)$ denote the number of conjugacy classes of $G$, and let

$$
1=\frac{1}{m_{1}}+\frac{1}{m_{2}}+\cdots+\frac{1}{m_{k}}
$$

be the centralizer equation of $G$, where if $1=x_{1}, x_{2}, \cdots, x_{k}$ are chosen from the $k$ conjugacy classes of $G$, then $m_{i}=\left|C_{G}\left(x_{i}\right)\right|$. We assume that the indexing is such that

$$
|G|=m_{1} \geqq m_{2} \geqq \cdots \geqq m_{k} .
$$

A finite group for which

$$
m_{1}>m_{2}>\cdots>m_{k}
$$

is called a repetition-free group. Repetition-free groups were first studied by Markel (1973); he proved that a supersolvable repetition-free group is necessarily isomorphic to Sym(3), the symmetric group on three symbols. Earlier Markel (1972) conjectured that Sym (3) is the only repetition-free group. In this paper we obtain a partial solution to Markel's conjecture. In fact, we prove the following:

(C) Copyright Australian Mathematical Society 1977

Copyright. Apart from any fair dealing for scholarly purposes as permitted under the Copyright Act, no part of this JOURNAL may be reproduced by any process without written permission from the Treasurer of the Australian Mathematical Society. 
THEOREM. If $G$ is a repetition-free group for which $m_{k} \leqq 4$, then $G \cong$ Sym (3).

\section{Proof of the theorem for $m_{k} \leqq 3$}

The following lemma summarizes the well-known properties of repetition-free groups which are required in the sequel. Parts (a) through (c) are easy to see, while Part (d) is proved in Markel (1972).

Lemma 2.1. Let $G$ be a repetition-free group.

(a) For $x \in G, x$ is conjugate to $x^{m}$ whenever $(m,|x|)=1$ and this power conjugate property is inherited by the homomorphic images of $G$.

(b) If some $m_{i}=p$, where $p$ is a prime and $i>1$, then $|G|$ is not divisible by $p^{2}$ and $p$ does not divide any other $m_{j}$ where $j>1$.

(c) If $p$ divides $|G|$, then $p-1$ also divides $|G|$.

(d) If $|G|=p^{\alpha} q^{\beta}$ where $p, q$ are distinct primes, then $G \cong \operatorname{Sym}(3)$.

REMARK. Recall that a (finite) group $G$ is said to be rational if it is power conjugate. Furthermore a group $G$ is rational if and only if every complex character of $G$ is rational-valued (see, for example, Huppert (1967), Satz V.13.7.b). Finally we note that the class of rational groups is closed under the operation of taking homomorphic images.

If $G$ is repetition-free and $m_{k}=2$, it follows easily from Burnside (1911, Note A, p. 462), that $G \cong \operatorname{Sym}(3)$.

Next we assume that $m_{k}=3$. Thus $G$ contains a self-centralizing element of order 3. So by a theorem of Feit \& Thompson (1962), one of the following statements must be true:

(a) $G$ contains a nilpotent normal subgroup $N$ such that $G / N \cong$ Alt (3) or Sym (3);

(b) $G$ contains a normal subgroup $H$ which is a 2 -group such that $G / H \cong$ Alt (5);

(c) $G \cong \operatorname{PSL}(2,7)$.

Now if $G / N \cong \operatorname{Sym}(3)$, where $N$ is nilpotent, we can see that $G$ cannot be repetition-free as follows:

Let $P$ be the set of primes which divide the order of $N$. Since $N$ is nilpotent, the center $Z(N)$ of $N$ is a $P$-group if and only if $N$ is a $P$-group. Now let $x \in Z(N)^{*}=Z(N) \backslash\{1\}$, then the order $|K(x)|$ of the conjugacy class of $x$ in $G$ divides $|\operatorname{Sym}(3)|$. Thus $|K(x)|=2,3$ or 6 for every $x$ in $Z(N)^{*}$. Since $Z(N) \triangleleft G, Z(N)$ is a union of complete sets of conjugacy classes of $G$. If $G$ is repetition-free, these classes must have distinct sizes and so $|Z(N)|=3$, $4,7,6,9,10$ or 12 . The case $|Z(N)|=3,6,9$ or 12 is impossible since $|G|$ is 
not divisible by 9 of Lemma 2.1(b). The case $|Z(N)|=4$ implies $|N|=2^{\alpha}$ and $|G|=2^{a+1} \cdot 3$. Thus $G$ is not repetition-free by Lemma 2.1(d). If $|Z(N)|=10$, then $Z(N)$, being Abelian, is a cyclic group of order 10 , and so $Z(N)$ contains exactly one element of order 2 . This, however, is incompatible with the partition of $Z(N)$ into three conjugacy classes of sizes 1,3 and 6 . Finally, suppose $|Z(N)|=7$. Then if $x$ is a generator of $Z(N)$, we have $|K(x)|=6$, and so $C_{G}(x)=N$. Hence

$$
\operatorname{Sym}(3) \cong G / N=N_{G i}(Z(N)) / C_{b i}(Z(N)) \leq \operatorname{Aut}(Z(N)) .
$$

This is impossible since Aut $(Z(N))$ is a cyclic group of order 6 .

In the other cases $G$ cannot be repetition-free since PSL (2.7), Alt (3) and Alt (5) are not rational.

\section{Proof of the theorem for $m_{k}=4$}

The proof in this case is carried out by means of a series of lemmas. We start with the following results of Wong (1967):

THEOREM A. Let $G$ be a finite group with a non-cyclic subgroup Tof order 4 which is its own centralizer in $G$. Then one of the following statements is true:

(1) $G \cong M_{11}$ or Alt (7);

(2) if $K$ is the largest normal subgroup of odd order in $G$, then $G / K$ is isomorphic with PSL $(3,3), G L(2,3), H(q)$ ( $q$ the square of an odd prime power), PGL $(2, q)$, PSL $(2, q)$ ( $q$ odd), or a 2-group of dihedral or semidihedral type.

THEOREM B. Let $G$ be a finite group with a cyclic subgroup $T$ of order 4 which is self-centralizing in $G$. If $K$ is the largest normal subgroup of odd order in $G$, then one of the following statements is true:

(1) $G \cong$ Alt (7);

(2) $K$ is Abelian and $G / K$ is isomorphic with $S L(2,3), S L(2,5)$, PSL $(2,7)$ or PSL $(2,9)$;

(3) the derived group $K^{\prime}$ of $K$ is nilpotent and $G / K$ is isomorphic with PGL (2,3), PGL (2,5). H(9), J, a 2-group of semi-dihedral or generalized quaternion type, a dihedral group of order 8, or a cyclic group of order 4.

The groups $M_{11}$, Alt (7), PSL $(3,3), \operatorname{GL}(2,3), \operatorname{PGL}(2, q)$ ( $q$ odd and $\neq 3,5), \operatorname{SL}(2,3), \operatorname{SL}(2,5), \operatorname{PSL}(2, q)$ ( $q$ odd), $H(q)$ ( $q$ the square of an odd prime power), $C_{4}$ and $J$ are not rational. Hence the corresponding $G$ cannot be repetition-free. The groups $\operatorname{PGL}(2,3)$ and $\operatorname{PGL}(2,5)$ are rational but not repetition-free, and it is easy to see that the corresponding $G$ cannot be repetition-free in these cases also. 
THEOREM 3.1. A 2-group $G$ of dihedral, semi-dihedral or generalized quaternion type is power conjugate only if it is either the ordinary dihedral group $D$ of order 8 , the ordinary quaternion group $Q$ of order 8 or the four-group $V$.

ProOf. The groups

$$
\begin{aligned}
& D_{\alpha}=\left\langle x, y \mid x^{2^{\alpha}}=1=y^{2}, y^{-1} x y=x^{-1}\right\rangle \\
& Q_{\alpha}=\left\langle x, y \mid x^{2^{\alpha}}=y^{2}=z, z^{2}=1, y^{\prime} x y=x^{-1}, \alpha \geqq 2\right\rangle
\end{aligned}
$$

and

$$
S D_{\alpha}=\left\langle x, y \mid x^{2^{\alpha}}=y^{2}=1, y^{-1} x y=x^{-1+2 \alpha \cdot 1}, \alpha \geqq 3\right\rangle
$$

are respectively the 2 -groups of dihedral, generalized quaternion and semidihedral type of order $2^{\alpha+1}$. So it suffices to show that $\alpha=1$ or 2 . Let $h$ be an element of maximal order in $G$. Then $|h|=2^{\circ}$. Every odd power of $h$ is conjugate to $h$ in $G$. Hence the automorphism $\phi: h \rightarrow h^{m}, m$ odd, is induced by an inner automorphism of $G$. Since $[G: C(h)]=2$ it follows that $\phi$ is a unique automorphism of order 2 and so the only admissible odd powers of $h$ are $h$ and $h^{-1}$. Hence $\alpha=1$ or 2 .

LEMMA 3.2. Let $G$ be a group with a self-centralizing cyclic subgroup of order 4 and $K$ the maximal normal subgroup of odd order in $G$. Suppose that the factor group $G / K$ is isomorphic to the quaternion group $Q$ of order 8 . Then $G$ is not repetition-free.

Proof. Suppose that $G$ is repetition-free and $G / K \cong Q=\langle x, y| x^{2}=y^{2}$, $\left.y^{-1} x y=x^{-1}\right\rangle$. Let $\langle x\rangle$ be self-centralizing in $G$, so that the corresponding $m$ is 4. Then since $y$ and $x y$ are not conjugates of $x$ we must have $m>4$ for them. Hence there is a non-identity element $u$ in $K$ so that $u$ centralizes $y$. So $\langle y u\rangle$ is a cyclic group of order $4 q$ where $u$ has order $q$. Since $(4 q, 4 q-1)=1$, $N_{G}(\langle y u\rangle) / C_{G}(\langle y u\rangle)$ must be isomorphic to the full automorphism group of $\langle y u\rangle$. Now $\left\{y^{-1}, u\right\}$ is a set of generators for $\langle y u\rangle$, hence the correspondence $y \rightarrow y^{-1}$ and $u \rightarrow u$ defines an automorphism of $\langle y u\rangle$. Hence there must be an element $a$ in $G$ (in fact, in $N_{G}(\langle y u\rangle)$ ) so that $a^{-1} y a=y^{-1}$ and $a^{-1} u a=u$. Thus $a \in C_{G}(u)$. Also we know that every element $k \in K$ can be written in the form $w^{-1} w^{x}$ for a suitable $w \in K$ and so every element of $x K \cup x^{1} K$ is conjugate to $x$. Hence $\left(x K \cup x^{-1} K\right) \cap C_{G}(u)=\varnothing$. Since $a^{-1} y a=y^{-1}$, a has even order and so $a \notin K$. If $a=y k$ for some $k \in K$ then $k^{-1} u k=u$ and $k^{-1} y k=y^{-1}$ and so as before $k$ cannot be in $K$. Hence we must have $a \in x y K \cup(x y)^{-1} K$. So, for some $k$ in $K, C_{G}(u)$ contains $x y k$ or $y^{-1} x^{-1} k$, and $y$. This implies that $x^{-1} k \in C_{G}(u)$ which is a contradiction.

LEMMA 3.3. Let $G$ be a group with a self-centralizing cyclic subgroup of 
order 4 and $K$ the maximal normal subgroup of odd order in $G$. Suppose that the factor group $G / K$ is isomorphic to the dihedral group $D$ of order 8. Then $G$ cannot be repetition-free.

Proof. If $G$ is repetition-free then $G / K^{\prime}$ is power-conjugate. In particular, every element of $K / K^{\prime}$ of prime order $p$ is conjugate to all its non-trivial powers under the action of $G / K$. It follows that $p-1=2$ or 4 , so $p=3$ or 5 . Furthermore, $K / K^{\prime}$ cannot have elements of order 9,15 or 25 , since otherwise $G / K$ would contain an Abelian group of order 6,8 or 20 . Hence $K / K^{\prime}$ is an elementary Abelian $p$-group where $p=3$ or 5 . Since $K$ admits a fixed-pointfree automorphism of order 4 , it follows that $K^{\prime}$ is nilpotent. Thus if $K^{\prime}$ is a $P$-group for some set $P$ of primes, then so is its center $Z\left(K^{\prime}\right)$. Let $x \in Z\left(K^{\prime}\right)$ be an element of prime order $q$. Since $K^{\prime}$ centralizes $x$, we have that the order of the factor group $N_{G}(\langle x\rangle) / C_{G}(\langle x\rangle)$ divides the index $\left|G: C_{G}(\langle x\rangle)\right|$ which in turn divides the order of $G / K^{\prime}$ and furthermore $N_{G}(\langle x\rangle) / C_{G}(\langle x\rangle) \cong$ Aut $(\langle x\rangle)$ is a cyclic group of order $q-1$. Hence

$$
q-1= \begin{cases}2,4 \text { or } 6 & \text { if } p=3 \\ 2,4 \text { or } 10 & \text { if } p=5\end{cases}
$$

and

$$
q= \begin{cases}3,5 \text { or } 7 & \text { if } p=3 \\ 3,5 \text { or } 11 & \text { if } p=5\end{cases}
$$

Now $Z\left(K^{\prime}\right)$ cannot contain elements of order 15,35 or 55 as this would lead to an Abelian group of order 8,24 or 40 which does not exist in $G$. Hence if $p=3, Z\left(K^{\prime}\right)$, and hence $K^{\prime}$, is a 5 -group, otherwise it is a $\{3,7\}$-group. We now consider the various cases in turn.

Case 1. $p=5$ and $\left|K^{\prime}\right|=5^{\alpha}$.

In this case $K$ is a 5 -group. Thus $|G|=8.5^{\beta}$. Hence $G$ is not repetition-free by Lemma 2.1.

Case 2. $p=5$ and $\left|K^{\prime}\right|=3^{\prime \prime} .11^{\delta}, \delta \neq 0$.

Here $|K|=S_{5} . S_{3} . S_{11}$, where no element of $S_{5}^{*}$ commutes with any element of $S_{3}^{*}$ or $S_{11}^{*}$, while every element of $S_{3}$ commutes with every element of $S_{11}$. If an $m_{i}=5$, then no other $m_{i}$ is 5 . Hence

$$
1<\frac{1}{4}+\frac{1}{8}+\frac{1}{5}+\left(1+\frac{1}{2}+\frac{1}{4}\right)\left(1+\frac{1}{3}+\frac{1}{9}+\cdots\right)\left(\frac{1}{11}+\frac{1}{121}+\cdots\right)<1,
$$

contradiction. On the other hand if no $m_{i}=5$, then we have 


$$
\begin{aligned}
1< & \frac{1}{4}+\frac{1}{8}+\left(\frac{1}{2}+\frac{1}{4}\right) \frac{1}{5}+\left(1+\frac{1}{2}+\frac{1}{4}\right)\left(\frac{1}{25}+\frac{1}{125}+\cdots\right) \\
& +\left(1+\frac{1}{2}+\frac{1}{4}\right)\left(\frac{1}{3}+\frac{1}{9}+\cdots\right)\left(\frac{1}{11}+\frac{1}{121}+\cdots\right) \\
< & 1 .
\end{aligned}
$$

Thus we cannot have a repetition-free group in this case.

Case 3. $p=3$ and $K^{\prime}$ is a 5-group, or $p=5$ and $K^{\prime}$ is a 3-group.

In this case $K=S_{3} . S_{5}$ and no element of $S_{3}^{*}$ commutes with any element of $S_{5}^{*}$, since there is no element of order 15. If $m_{i}=5$ occurs, then no other $m_{i}$ is divisible by 5 . Also recall that there is no element of order 12 . Hence

$$
1<\frac{1}{4}+\frac{1}{8}+\left(\frac{1}{9}+\frac{1}{27}+\cdots\right)+\frac{1}{2}\left(\frac{1}{3}+\frac{1}{9}+\cdots\right)+\frac{1}{5}<1 .
$$

Whereas if no $m_{i}=5$ we get

$$
\begin{aligned}
1< & \frac{1}{4}+\frac{1}{8}+\left(\frac{1}{9}+\frac{1}{27}+\cdots\right)+\frac{1}{2}\left(\frac{1}{3}+\frac{1}{9}+\cdots\right) \\
& +\left(1+\frac{1}{2}\right)\left(\frac{1}{5}+\frac{1}{25}+\cdots\right)-\frac{1}{5}<1,
\end{aligned}
$$

since there is no element of order 20. So in both subcases we get a contradiction.

Case 4. $\left|K^{\prime}\right|=3^{\alpha}$ and $p=3$.

$G$ cannot be repetition-free by Lemma 2.1 .

Final case: $p=3$ and $K^{\prime}$ is a $\{3,7\}$-group.

Consider the action of the elementary Abelian group $K / K^{\prime}$ on $Z\left(S_{7}\right)$. Then by a theorem of Wielandt (1960), as long as $\left|K / K^{\prime}\right|>3$ there exists an element $u_{1} \in K / K^{\prime}$ so that $u_{1}$ leaves an element in $S_{7}^{(1)}=Z\left(S_{7}\right)$ fixed. Let $K_{2}=\left\langle K^{\prime}, u_{1}\right\rangle$, then $Z\left(K_{2}\right) \cap S_{7} \neq\langle 1\rangle$. Now consider the action of $K / K_{2}$ on $Z\left(K_{2}\right) \cap S_{7}=S_{7}^{(2)}$. If $\left|K / K_{2}\right|>3$ there exists a $u_{2} \in K / K_{2}$ which leaves some element of $S_{7}^{(2)}$ fixed. So $K_{3}=\left\langle u_{2}, K_{2}\right\rangle$ satisfies $Z\left(K_{3}\right) \cap S_{7}^{(3)} \neq\langle 1\rangle$. Repeating this we get to $K_{\beta-1}$ with $\left|K / K_{\beta-1}\right|=3$ and $Z\left(K_{\beta-1}\right) \geqq S_{7}^{(\beta-1)} \neq\langle 1\rangle$. Now since the centralizer of every element of $S_{7}^{(\beta-1)}$ has index less than or equal to 24 we cannot have $\left|S_{7}^{(\boldsymbol{\beta}-1)}\right|>7$ (that is $\geqq 49$ ), since the only conjugate classes of elements in $S_{7}^{(\beta-1)}$ have sizes $1,6,12$ or 24 and $1+6+12+24<49$. Hence there exists $z \in Z\left(S_{7}\right)$ which has either 6,12 or 24 conjugates.

If $z$ has six conjugates, then $G$ contains a characteristic subgroup of order 7 whose centralizer is a normal subgroup $H$ of index 6 , and $G / H$ is cyclic of order 6 , which is not power conjugate. Hence $G$ is not repetition-free by Lemma 2.1 . 
If $z$ has 12 conjugates, let the conjugates be $z, z^{2}, \cdots, z^{6}$ and $z^{\prime}, z^{\prime 2}, \cdots, z^{\prime 6}$ where $z^{\prime} \in Z\left(S_{7}\right)$ (a characteristic subgroup of $G$ ). The group $N=\left\langle z, z^{\prime}\right\rangle$ is elementary Abelian of order 49 and there is a homomorphism of $G$ into the group of automorphisms of $N$, in fact into that group $A$ (of order 72) which leaves the set $\left\{z^{\alpha}, z^{\prime \beta} \mid \alpha, \beta=0,1, \cdots, 6\right\}$ fixed. Let us use the additive notation, so we can represent the automorphisms as elements of GL $(2,7)$ :

$$
A=\left\{\left(\begin{array}{cc}
\alpha & 0 \\
0 & \beta
\end{array}\right),\left(\begin{array}{cc}
0 & \alpha \\
\beta & 0
\end{array}\right) \mid \alpha \beta \neq 0 ; \alpha, \beta \in \mathrm{GF}(7)\right\}
$$

The elements of $S_{2}(A)$ are

$$
\begin{aligned}
& \left(\begin{array}{ll}
1 & 0 \\
0 & 1
\end{array}\right)=1, \quad\left(\begin{array}{rr}
0 & -1 \\
1 & 0
\end{array}\right)=x, \quad\left(\begin{array}{rr}
-1 & 0 \\
0 & -1
\end{array}\right)=x^{2}, \quad\left(\begin{array}{rr}
0 & 1 \\
-1 & 0
\end{array}\right)=x^{3}, \\
& \left(\begin{array}{rr}
-1 & 0 \\
0 & 1
\end{array}\right)=y, \quad\left(\begin{array}{rr}
1 & 0 \\
0 & -1
\end{array}\right)=x^{2} y, \quad\left(\begin{array}{ll}
0 & 1 \\
1 & 0
\end{array}\right)=y x, \quad\left(\begin{array}{rr}
0 & -1 \\
-1 & 0
\end{array}\right)=y x^{3}
\end{aligned}
$$

which is isomorphic to $D$.

One group $S_{3}(A)$ is given by

$$
\begin{aligned}
& \left(\begin{array}{ll}
1 & 0 \\
0 & 1
\end{array}\right)=1, \quad\left(\begin{array}{ll}
2 & 0 \\
0 & 1
\end{array}\right)=u, \quad\left(\begin{array}{ll}
4 & 0 \\
0 & 1
\end{array}\right)=u^{2}, \quad\left(\begin{array}{ll}
1 & 0 \\
0 & 2
\end{array}\right)=v, \\
& \left(\begin{array}{ll}
1 & 0 \\
0 & 4
\end{array}\right)=v^{2}, \quad\left(\begin{array}{ll}
2 & 0 \\
0 & 2
\end{array}\right)=u v, \quad\left(\begin{array}{ll}
4 & 0 \\
0 & 2
\end{array}\right)=u^{2} v, \\
& \left(\begin{array}{ll}
2 & 0 \\
0 & 4
\end{array}\right)=u v^{2}, \quad\left(\begin{array}{ll}
4 & 0 \\
0 & 4
\end{array}\right)=u^{2} v^{2} .
\end{aligned}
$$

This is a normal subgroup of $A$ and commutes with the diagonal elements $1, x^{2}, y, x^{2} y$ of $S_{2}(A)$. The elements $u v, u^{2} v^{2}$ are in the center of $A$ and are not conjugates of each other, neither are the elements $u, u^{2}$ or $v, v^{2}$ conjugates of each other. Thus if we let $H=C(N)$ then $G / H \cong A_{1} \leqq A$ where on the one hand 12 divides $\left|A_{1}\right|$ (since $A_{1}$ acts transitively on the conjugacy class of $z$ ) and 3 does not divide $\left|A_{1}\right|$, since otherwise there would be an element of order 3 not conjugate to its inverse. Contradiction.

Finally, let $z \in Z\left(S_{7}\right)$ have 24 conjugates. Then the conjugates of $z$ generate a characteristic subgroup $N \leqq Z\left(S_{7}\right)$ of $G$ with $|N|=7^{2}, 7^{3}$ or $7^{4}$. The case $|N|=7^{2}$ we have settled already because the other conjugacy classes in $N$ must then have sizes 6 and $18(49=1+6+18+24)$ and the class of size 6 leads to the normal subgroup $N_{0} \leqq N,\left|N_{0}\right|=7$ whose centralizer has index 6 in $G$.

The case $|N|=7^{3}$ would lead to a decomposition of $N$ into conjugacy classes of sizes 1 and $6 s_{i}$ where $6\left(4+s_{1}+s_{2}+\cdots\right)=6 \cdot\left(7^{3}-1\right) / 6=6.57$ and 
there is no way of expressing 53 as a sum of distinct integers of the form $2^{\alpha} \cdot 3^{\beta}$ $(\neq 4), \alpha \leqq 2$, since the only choices for $s_{i}$ are $36,27,18,12,9,6,3$.

This leaves the case $|N|=7^{4}$. If we write the group additively we can express it in terms of a basis $z_{1}=z, z_{2}, z_{3}, z_{4}$ where the conjugacy class of $z$ is

$$
\left\{\alpha_{i} z_{i} \mid i=1,2,3,4 ; \alpha_{i}=1,2, \cdots 6\right\} \text {. }
$$

The group $A$ of conjugacies is isomorphic to a subgroup $B$ of $\mathrm{GL}(4,7)$ which leaves the conjugacy class of $z$ invariant. That is, $B$ is the group generated by the diagonal matrices and the permutation matrices which has order $6^{4} .24$. Since it is a homomorphic image of $G / K^{\prime}$ we know that $|A|$ cannot be divisible by a power of 2 higher than 8 and the Sylow 3-subgroup $S_{3}(A)$ is elementary Abelian. Since $S_{3}(B)$ is non-Abelian we have $S_{3}(B)>S_{3}(A)$ and therefore $|A|$ divides $8.3^{4}$.

Now we must express $7^{4}$ as the sum of orders of different conjugacy classes of orders 1 and $6 s_{i}$ where $s_{i} / 108$,

$$
\frac{7^{4}-1}{6}=4+s_{1}+s_{2}+\cdots, \quad s_{i} \mid 108, \quad s_{i} \neq 4
$$

so $s_{i} \in\{108,54,36,27,18,12,9,6,3\}$ and $4+\Sigma s_{i} \leqq 277<400$.

LEMMA 3.4. Let $G$ be a group of order $n$ which contains an element $x$ so that the conjugacy class $K(x)$ of $x$ in $G$ has $n / 4$ elements, where the centralizer of $x$ in $G$ is a four-group $V$. Then $G$ is not repetition-free or else $G$ has a self-centralizing cyclic subgroup of order 4.

Proof. Let $G$ be as in the hypothesis of the lemma. Then by Theorem A either

(a) $G \cong M_{11}$ or Alt (7)

or

(b) if $N$ is the maximal normal subgroup of odd order in $G$, then $G / N$ is isomorphic to PSL (3,3), GL (2,3), PGL $(2, q), \operatorname{PSL}(2, q)$ ( $q$ odd); $H(q)$ ( $q$ the square of an odd prime power), or a 2-group of dihedral or semi-dihedral type.

All these cases have been dealt with except when $G / N$ is isomorphic to a 2-group of dihedral or semi-dihedral type. However by our reduction Theorem 3.1, we know that if $G$ is also repetition-free, then we have

(i) $G / N \cong V$ (the four group), or

(ii) $G / N \cong D$ (the dihedral group of order 8 ).

In the first case, we have $x N \in V$ and consider $H=\langle x, N\rangle$. Since $x$ does not commute with any element of $N$ (except the identity) it follows that the conjugacy class of $x$ still has $|N|=|H| / 2$ elements. Thus all the other 
conjugacy classes have size 2 (except the identity). This means that $N$ is Abelian, and the only possible sizes of conjugacy classes of $G$ whose elements are in $N$ are 1,2,4 so either $|N|=3,5$ or 7 , none of which works (just by adding the $1 / m_{i}$ ).

In case (ii), we have $x N \in D$ and $H=\langle x, N\rangle$ is a group in which $x$ has $|N|$ distinct conjugates. Thus either $|K(x)|=|H| / 2$ and we have $N$ Abelian as before, or $|K(x)|=|H| / 4$ and $x$ generates a cyclic group of order 4 which is self-centralizing in $H$.

\section{Acknowledgement}

The author wishes to thank the referee for helpful suggestions during the preparation of this paper.

\section{References}

W. Burnside (1911), Theory of Groups of Finite Order, 2nd ed. (Dover, New York, 1955).

Walter Feit and John G. Thompson (1962). 'Finite groups which contain a self-centralizing subgroup of order 3', Nagoya Math. J. 21, 185-197.

B. Huppert (1967). Endliche Gruppen I, (Die Grundlehren der mathematischen Wissenschaften in Einzeldarstellungen Band 134, Springer-Verlag, Berlin, Heidelberg, New York. 1967).

Frank M. Markel (1972), Conjugacy class problems for finite groups (PhD thesis, University of Toronto, Ontario, 1972).

Frank M. Markel (1973), 'Groups with many conjugate elements', J. Algebra 26. 69-74.

Helmut Wielandt (1960), 'Beziehungen zwischen den Fixpunktzahlen von Automorphismengruppen einer endlichen Gruppe', Math. Z. 73, 146-158.

Warren J. Wong (1967), 'Finite groups with a self-centralizing subgroup of order 4', J. Austral Math. Soc. 7, 570-576.

Department of Mathematics,

University of Ife,

ILE-IFE, Nigeria. 